\title{
Les universités françaises au milieu du gué
}

\author{
Pierre Cornu*
}

Histoire contemporaine et histoire des sciences, Université Lyon 2, EA Laboratoire d'études rurales, Lyon, France

\begin{abstract}
Résumé - Depuis le lancement il y a plus de vingt ans de la politique d'harmonisation et d'intégration de l'enseignement supérieur et de la recherche à l'échelle européenne, les universités françaises sont entrées dans un processus continu de réformes qui a certes puissamment contribué à les ouvrir et à les connecter à l'économie globale de la connaissance, mais qui les a également fragilisées et divisées, remettant en cause leur aptitude à contribuer aux défis scientifiques et éducatifs posés par le changement global. Trop exclusivement considéré du point de vue de ses enjeux institutionnels et budgétaires, ce processus d'extraversion du modèle universitaire français doit être restitué dans ses enjeux épistémologiques et dans son inscription dans l'histoire de l'idée d'université, pour permettre une sortie par le haut des crispations actuelles sur le modèle de l'excellence académique.
\end{abstract}

Mots-clés : universités / politique de la recherche et de l'enseignement supérieur / excellence scientifique / économie de la connaissance / relations sciences-sociétés / interdisciplinarité / épistémologie historique

\begin{abstract}
French universities in midstream. Since the political process of European harmonization and integration of higher education and research institutions was launched more than twenty years back, French universities have entered a continuing reform process which on the one hand has strongly contributed to opening and connecting them to the global knowledge economy, while on the other hand weakening and dividing them, thus threatening their ability to contribute to the scientific and educational challenges raised by global change. Overly focused on institutional and budgetary aspects, the extroversion process of the French academic landscape must be reappraised in its epistemological dimension, as well as from the historic perspective of the concept of university, allowing us to consider a positive way out of the current tensions on the topic of academic excellence.
\end{abstract}

Keywords: universities / politics of research and higher education / scientific excellence / knowledge economy / sciences-society relationships / multidisciplinarity / historical epistemology

Depuis le lancement en 1997 à Lisbonne du processus d'harmonisation et d'intégration de l'enseignement supérieur et de la recherche à l'échelle européenne, selon une ligne libérale jamais démentie depuis, et même réaffirmée avec une force croissante de conférence en conférence, les choses semblaient claires pour les universités françaises : certes peu enclines à adopter un modèle éloigné de leur culture et mal préparées à l'«autonomie», mais averties par l'absence quasi totale d'impact sur le processus de réforme des alternances politiques et de la crise du projet européen dans les années 2000, elles savaient n'avoir pas d'autre espoir que de retarder l'avènement ou de gagner des garanties à la marge du paradigme de l'excellence compétitive. C'est ainsi par une alternance d'usage tactique

\footnotetext{
*Auteur correspondant : pierre.cornu@univ-lyon2.fr
}

de la force d'inertie dans les moments de faiblesse ou de transition politiques, et de moments de zèle plus ou moins sincère, destinés à saisir les opportunités offertes par les dispositifs de soutien à l'extraversion de l'enseignement supérieur et de la recherche, notamment la succession des «programmes investissements d'avenir» (PIA), que les universités entrèrent cahin-caha dans la voie des fusions, des fédérations et des mises en réseaux. En pleine conscience des enjeux institutionnels, budgétaires et politiques du processus, mais par trop aveugles sur ses implications épistémologiques et sur les menaces qu'il fait peser sur les relations entre sciences et sociétés. C'est du moins ce que nous voudrions développer ici, non pour ajouter une crise à la crise, mais pour suggérer une voie de résolution qui mobilise ce que l'idée d'université peut receler encore de ressources, dans sa déclinaison nationale et européenne, pour porter l'entreprise du logos à la hauteur des défis du temps. 


\section{L'autonomie, schème directeur ambivalent}

Si les établissements français ne furent jamais égaux dans leurs capacités à développer leurs missions, même au temps où la continuité territoriale était effectivement un principe cardinal des politiques publiques, l'effet du passage à l'autonomie budgétaire, couplé à la mise en œuvre de dispositifs d'excellence sur concours arbitrés par des jurys internationaux - donc, sans souci prioritaire de l'aménagement du territoire -, fut inévitablement de creuser des écarts importants entre les établissements, comme le soulignent Xavier Arnauld de Sartre et Olivier Petit dans l'éditorial de ce numéro de $N S S^{1}$. Très rapidement, cela mit en jeu la soutenabilité de l'offre de formation et de l'engagement dans la recherche des moins bien lotis, tout d'abord dans le monde des sciences humaines et sociales, puis également dans les disciplines scientifiques les moins connectées à la demande privée, et, toutes disciplines confondues, dans les établissements les plus récents et les plus modestes en taille, nés du grand élan de la décentralisation des années 1980 et 1990. Même du côté des sites les mieux dotés toutefois, le processus d'intégration et d'extraversion $s$ 'avéra particulièrement périlleux, notamment du fait des équilibres délicats à trouver avec les grands opérateurs de recherche (CNRS, Inserm, Inra...), peu adeptes du «localisme», fût-il "métropolitain», et plus encore avec les grandes écoles, normales et ingénieriales, inquiètes d'une dilution de leurs forces et de leurs identités dans des entités ingouvernables. Obtenu de haute lutte, le label Idex s'avéra parfois destructeur des fragiles synergies locales, comme à Toulouse, où la tentative de produire un saut qualitatif collectif révéla un dissensus fondamental sur le sens que les uns et les autres donnaient au concept d'《excellence».

De fait, un petit nombre d'établissements universitaires seulement se révélèrent capables de jouer le jeu de la montée en capacité et du développement des interfaces à la fois à l'échelle des sites et à celle des réseaux internationaux, tandis que beaucoup vécurent le passage à l'autonomie comme un purgatoire, générant de fortes tensions internes, une instabilité de leur gouvernance, et une mise entre parenthèses de tout projet propre - rendant particulièrement ironique l'injonction à participer aux concours de labellisation. Même un cas de fusion réussie comme Clermont-Ferrand, avec constitution d'un établissement unique et obtention du label «I-Site » à la deuxième tentative en 2017, a laissé un goût amer à une partie des enseignants-chercheurs de la capitale auvergnate, contraints

\footnotetext{
${ }^{1}$ Arnauld de Sartre X., Petit O., 2018. Une nouvelle géographie de l'enseignement supérieur et de la recherche: rapprochements, concentrations, tensions... et opportunités?, Natures Sciences Sociétés, 26, 3.
}

de réduire leurs ambitions scientifiques comme leur offre pédagogique pour rentrer dans l'épure. C'est en effet le paradoxe du «passage à l'autonomie » des établissements que de les avoir forcés à sacrifier en premier lieu ce qui est le plus vital dans la recherche et dans la formation par la recherche: l'investissement humain et financier de moyen terme dans l'innovation méthodologique et didactique, clé du renouvellement de disciplines et de formations périodiquement émoussées dans leur pertinence heuristique, et instrument privilégié de la différenciation du service public par rapport à la «recherche et développement » privée. Comment dès lors s'étonner que l'autonomie ait produit plus de Machiavels que de Léonards?

\section{Tensions gestionnaires et crises politiques}

Tiraillés entre la puissance des injonctions descendantes et les inquiétudes, voire la colère, de leurs personnels et de leurs étudiants, de moins en moins canalisées par les syndicats traditionnels, bien des présidents d'université, confrontés à des alternances difficiles en 2012 et en 2016, et parfois même à des accidents de gouvernance en cours de mandat, se sont de fait trouvés amenés à abandonner leurs professions de foi quand il a fallu affronter les conséquences des évaluations nationales, des discussions budgétaires avec l'État ou de la mise en œuvre des étapes du programme investissement d'avenir et de ses déclinaisons successives. Conçues pour les chemins larges et rectilignes des plaines sédimentaires du positivisme, les institutions universitaires se sont avérées des attelages bien peu maniables dans les reliefs tourmentés de la «science $2.0^{2} »$, et les enseignants-chercheurs qui en tenaient les rênes, des conducteurs bien mal préparés à l'exercice de la gouvernance de crise. Lorsqu'il deviendra possible de faire une prosopographie des exécutifs d'universités des années 2000 et 2010, on trouvera sans doute davantage d'histoire de ruptures, parfois douloureuses, entre les individus et leurs mondes académiques d'origine, que d'ascensions maîtrisées vers les responsabilités. Ce sera, il faut le craindre, sans fleurs ni couronnes pour les vaincus, et sans confiance ni reconnaissance pour les vainqueurs, notamment auprès de la masse des maitres de conférences et des vacataires, épuisés et fragilisés par la maintenance de dispositifs de premier cycle universitaires de moins en moins adaptés aux mutations de la société comme de l'économie de la formation et de la connaissance. C'est en effet une autre conséquence de la complexification institutionnelle que d'avoir produit une rupture dans les carrières entre « soutiers » et habitués du mess des officiers, non pas sur des critères admis de valeur scientifique,

\footnotetext{
${ }^{2}$ Nowotny H., Scott P., Gibbons M., 2001. Re-thinking science. Knowledge and the public in an age of uncertainty, Cambridge, Polity Press.
} 
d'ancienneté ou de représentation corporatiste, ni même dans une reproduction du modèle ancien du «mandarinat », mais sur des logiques d'acculturation managériale, avec le risque que cette nouvelle voie de sélection des «cadres» n'ait des effets normatifs accentués sur l'évaluation de la recherche et des formations, notamment en termes d'interdisciplinarité et de soutien aux questionnements émergents. Non que les instruments du libéralisme ne sachent encourager l'innovation; mais justement, parce qu'ils sont pensés pour des modèles descendants et directifs de production de l'innovation qui contreviennent à la logique itérative de beaucoup de disciplines de la recherche publique.

Si la Conférence des présidents d'université (CPU) a fait son possible, depuis les grands mouvements de protestation de la présidence Sarkozy, pour mutualiser les risques et les opportunités de l'interface entre communautés universitaires et politiques publiques, y gagnant une visibilité et une légitimité renforcées, elle n'a de fait pas pu trancher les dilemmes auxquels se trouvaient confrontés les responsables d'exécutifs locaux entre «bonne gestion» et valeurs scientifiques et pédagogiques, quand ce n'était pas entre chances de survie de leur établissement et respect des règles et des procédures de la démocratie universitaire. Là encore, il faudra attendre pour analyser les archives des conseils d'administration de cette période agitée, mais on risque d'y lire davantage de discours de rupture, voire d'incompréhension franche et brutale, que de témoignages d'un quelconque esprit de conciliation-avec des dégâts non négligeables pour l'image globale des universités, aux yeux notamment des «membres extérieurs » des conseils et, plus largement, des partenaires socio-économiques et culturels de l'enseignement supérieur et de la recherche.

\section{Les anamorphoses de la carte académique française}

En l'espace de vingt ans, la carte de l'enseignement supérieur et de la recherche en France (Fig. 1) s'est ainsi trouvée profondément bouleversée, avec l'émergence rapide d'établissements de rang européen (guère encore de rang mondial) dans les métropoles régionales les plus adaptées au modèle, Strasbourg représentant aussi bien pour ses laudateurs que pour ses détracteurs l'idéal-type de l'université intégrée et efficacement extravertie. Certes, à Paris, la situation est encore loin d'être décantée, en raison de la densité du système académique développé dans la capitale et à sa périphérie, complexifié encore par les opérations de transferts de Paris intra muros vers le plateau de Saclay ou le Campus Condorcet de certains fleurons de l'enseignement supérieur. Mais c'est peut-être le sort de l'EHESS, qui a dû affronter à ce jour un nombre invraisemblable de scénarios d'association, qui illustre le mieux le caractère kafkaïen du processus à l'œuvre en Île-de-France-surtout si l'on considère l'histoire propre de cette école, jadis symbole de l'ouverture interdisciplinaire et internationale des sciences sociales françaises... Les problèmes de «riches» (très relatifs) des grands établissements de la capitale ne doivent d'ailleurs pas occulter ceux des territoires académiques caractérisés par la dispersion des universités et de leurs "antennes», comme dans le Grand Ouest, menacés d'invisibilisation par les effets de seuil du modèle "grand établissement intégré », quand bien même, si l'on se fie, justement, aux critères de l'excellence scientifique et de l'originalité des formations, certains pôles de taille modeste, comme Pau, n'ont pas à rougir de leur offre. Certes, on ne peut espérer refonder l'université française sur l'utopie d'une abolition de la compétition pour l'excellence scientifique, négation même de l'élan qui anime la recherche et la formation par la recherche. Mais on peut, de fait, contester la pertinence d'une hiérarchie des établissements fondée sur les contingences de la géographie académique.

$\mathrm{Au}$ reste, on ne saurait résumer les trajectoires des universités françaises dans la réforme européenne aux seuls critères de la taille et du fait métropolitain, comme l'illustrent les exemples contrastés de Bordeaux, Lyon et Grenoble, a priori également doués pour passer avec succès le test de l'excellence et de la taille critique. Fusion avec dissidence, fusion inaboutie, fusion complète avec subsidiarité entre les échelles de la gouvernance, les transitions de ces sites vers l'intégration internationale racontent des histoires singulières, qui mettent l'observateur sur la piste d'une réalité trop souvent occultée dans les débats sur la gouvernance de l'enseignement supérieur et de la recherche : le fait que, pour la plupart d'entre eux, les établissements européens s'inscrivent dans une histoire pluriséculaire, et que, certes, les processus à l'œuvre dans le monde de la globalisation de la compétition scientifique constituent un défi inédit aux « vieilles maisons » du continent, mais que pour autant, leur mémoire reste vivace et leur potentiel autrement plus riche que leur «bilan RH», l'état de leurs finances et leurs ressources immobilières ne le laisseraient penser. Traditions humanistes, saint-simoniennes, technicistes, interculturelles, girondines ou jacobines, ne se trouvent pas également réparties et hybridées, et commandent une intelligence fine des écosystèmes académiques. Marseille et Brestn'ont pas le même rapport à la mer, Limoges et Nancy ne dialoguent pas de la même manière avec leur territoire d'ancrage. Et pardelà même ces idiosyncrasies locales, les établissements abritent des communautés fort anciennes et profondément singulières dans leurs chartes fondatrices, comme le révèlent les modes assez distincts de sanction des carrières des différentes sections du Conseil national des universités (CNU). Traditions de conseil ou de contestation du prince, d'ouverture sur les arts ou sur les industries, d'ancrage dans la notabilité ou dans les contre-cultures, constituent ainsi un 


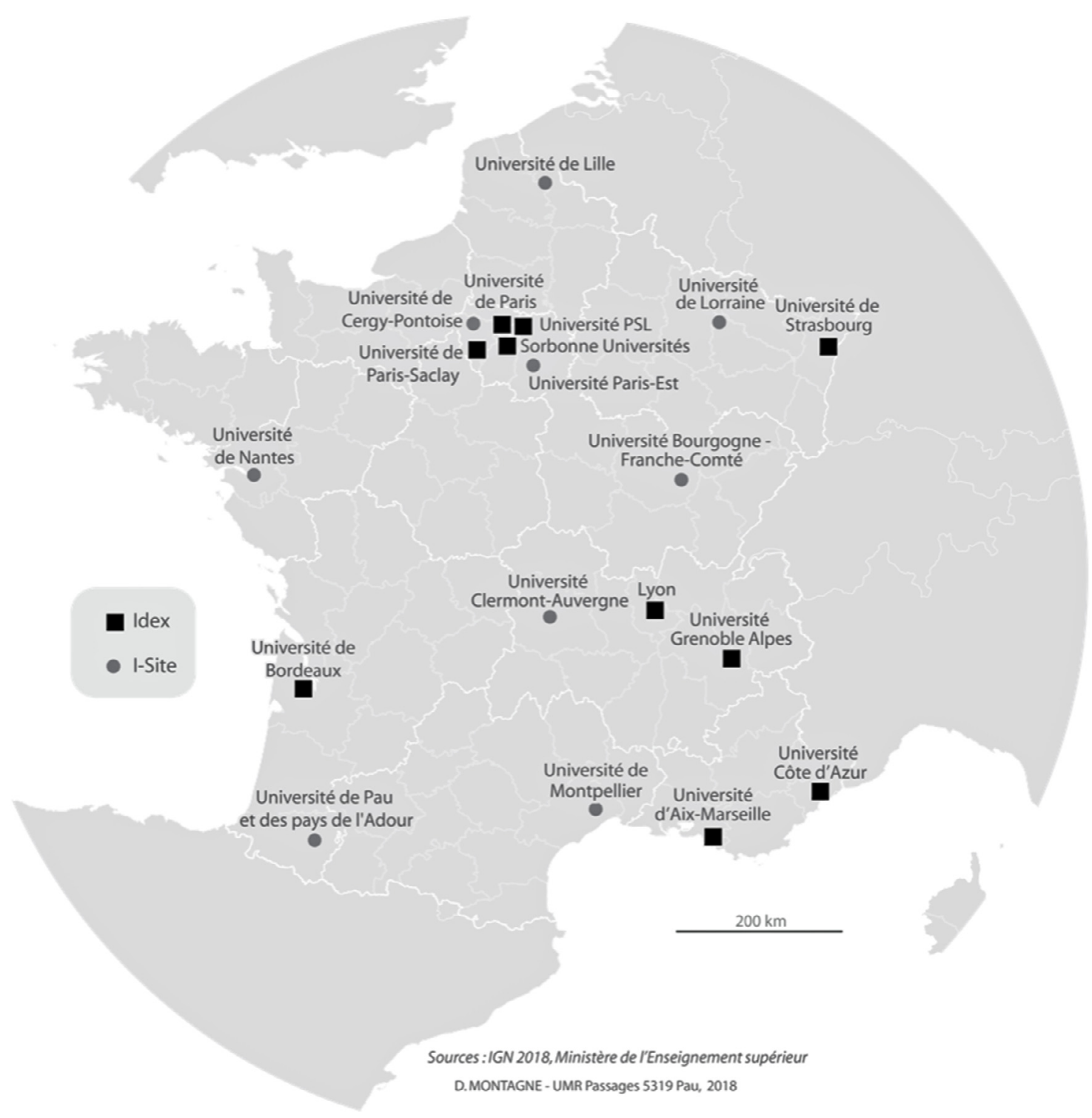

Fig. 1. Une géographie universitaire bouleversée par les labels d'excellence.

ethos universitaire aux mille nuances que l'on ne saurait rayer d'un trait de plume par simple souci de «modernisation ». Et peut-être faut-il chercher dans ce malentendu, davantage que dans un quelconque antimodernisme atavique, pourquoi les «libertés universitaires» se laissent si mal dissoudre dans la «libéralisation» de l'économie du savoir. Mal faits pour la massification, les établissements le sont tout autant pour la standardisation de leur production cognitive, et il est vraiment désolant que la crispation des débats sur le concept $d$ ' ' excellence» lui aliène un grand nombre d'enseignants-chercheurs, quand ce devrait être leur idéal propre que de voir la qualité distinctive de leur recherche et de leurs enseignements reconnus selon des grilles d'évaluation appropriées.

\section{De la crise des cultures académiques à la refondation des éthiques de l'engagement}

L'intensité de la remise en cause des habitus académiques par les politiques publiques n'a pas seulement eu pour effet de remobiliser des communautés professionnelles autour de banderoles aux couleurs ravivées, comme on l'a vu au printemps 2018, elle a également réveillé des schèmes «cléricaux» enfouis depuis longtemps, notamment celui de l'instrumentalisation des savoirs ou des rhétoriques de l'«intérêt général », de la «valeur» ou du «juste», menaçant de 
faire voler en éclats le dialogue entre le «savant» et le «politique». À cela, une explication trop rarement mobilisée: le contraste douloureux entre la plasticité croissante des institutions universitaires et la rigidité persistante des carrières. En effet, bientôt vingt ans de malthusianisme dans les recrutements ont généré des entraves aussi bien à la mobilité géographique qu'au changement de grade, interdisant la recomposition des communautés sur la logique de projets partagés, hormis dans quelques îlots privilégiés de «top academics». C'est ainsi que, dans les établissements, et plus sensiblement encore à l'échelle des facultés, les conflits inter-individuels et de conception de l'Universitas ne se décantent plus ni dans des prises de pouvoir ni dans des mutations, exacerbant les tensions et paralysant aussi bien la vie scientifique que l'innovation pédagogique.

Quoi qu'il en soit des blessures individuelles et collectives, et des conséquences institutionnelles et scientifiques de ce processus de réforme à marche forcée, on ne peut cependant que constater son efficacité politique et, pardelà des moments d'inquiétude collective sur les conditions de l'accès à l'enseignement supérieur, comme lors de la mise en place du dispositif d'affectation «Parcoursup » début 2018, son acceptation assez large par l'opinion publique. Il faut se faire une raison, les tourments de l'enseignantchercheur ne suscitent guère d'empathie, et les cassandres d'amphithéâtre n'ont pas l'efficacité des idéologues télévisuels pour orienter le débat public. Et l'on doit voir comme un symptôme alarmant de la déréliction du monde universitaire la tentation, chez certains, de sacrifier les codes de la scientificité à la production (et à la reproduction par le recrutement) d'un entre-soi militant, que celui-ci soit fondé sur une introversion corporatiste, sur une extraversion contestataire ou sur un alliage instable des deux. Pour les sciences sociales notamment, la rechute dans ce que l'historien appellerait volontiers l'habitus des «ordres mendiants »-ici franciscains, là dominicains... - représente à la fois une fragilité bien réelle, et une menace préoccupante. Comment ne pas voir en effet que le discours anticapitaliste et antilibéral qui transpire des murs de certains établissements, quelle que soit la valeur intrinsèque de ses arguments, ne fait que nourrir la perte en rationalité des sociétés contemporaines, pour parler comme Hermann Broch ${ }^{3}$, justifiant aux yeux d'une opinion déboussolée le sacrifice de l'expertise et de l'intelligence de la complexité, au profit d'une économie politique frelatée faisant commerce de surcompensations symboliques? Quand une partie du monde scientifique luimême doute du logos, et se convertit à la rhétorique du dolorisme subalterniste, c'est que la crise va au-delà de la relation entre l'État et ses chercheurs, qu'elle traduit un

\footnotetext{
${ }^{3}$ Broch H., 2008. Théorie de la folie des masses, Paris, Éditions de l'éclat (manuscrit publié à titre posthume, rédigé entre la fin des années 1930 et la fin des années 1940).
}

déphasage entre les représentations, les pratiques et les enjeux d'un monde où les technosciences et les dispositifs de gouvernement des êtres et des choses ne répondent plus à l'éthique ancienne du progrès.

On dira que l'université française présentait dès avant le lancement du processus d'intégration européenne une nette tendance à privilégier la production théorique, et de faibles capacités de mobilisation et de portage collectif de l'innovation. Certes. Mais n'était-ce pas l'esprit des réformes européennes, justement, que de développer un tropisme constructiviste dans les établissements ? Et à la lumière de cet idéal, y a-t-il lieu d'être satisfait du résultat et de ce qu'il a coûté, notamment sur les jointures les plus fragiles de l'édifice des savoirs, celles qui, justement, en assurent l'interactivité et la réflexivité ? Il est bien entendu trop tôt pour faire l'histoire et encore moins le bilan de ce processus, des monographies d'établissements et de sites restant à constituer, non pas seulement en termes statistiques et techniques, mais également en prenant en compte les aspects culturels et politiques des histoires académiques. Les atermoiements des établissements lyonnais, par exemple, y trouveraient des explications plus pertinentes que l'effet des brumes fluviales sur la clairvoyance politique des acteurs locaux, et peut-être des solutions nouvelles pour intégrer sans les brusquer les traditions humaniste, industrialiste et chrétienne-sociale de la capitale des Gaules. À Lyon comme ailleurs, il apparaît surtout particulièrement urgent de traiter les tensions à l'œuvre au sein même du monde de la recherche et de l'enseignement supérieur, celles qui menacent le dialogue entre sciences sociales, humanités, ingénieries et sciences de la nature, mais également les enjeux sciencessociété et, plus profondément, le potentiel des universités à contribuer à une économie de la connaissance réflexive et responsable.

\section{Crise des universités et réflexivité historique}

C'est en effet une conséquence paradoxale des rapprochements à marche forcée entre établissements que de créer des frictions sur les interfaces disciplinaires et épistémologiques, ravivant des oppositions que l'on pouvait penser en voie d'effacement entre sciences et savoirs se représentant comme « fondamentaux », et pratiques de l'enseignement et de la recherche assumant une quête de performativité au sein des systèmes sociaux, techniques ou biologiques. De fait, la carte des «points chauds» de la réforme des universités révèle non seulement des cas d'établissements en situation de stress insupportable entre moyens disponibles et exigences de service public, ou de crises générées par des rapports de force entre communautés académiques perçus comme déséquilibrés, mais également et plus profondément, des tendances schismatiques sur le sens et les missions de l'enseignement supérieur et de la recherche dans le 
monde du «changement global». Dans beaucoup de départements de sciences sociales, mais également de sciences de la nature, on observe une tentation d'un déni de toute responsabilité dans le devenir bioéconomique du monde et d'un repli sur des postures critiques exacerbées, invoquant un âge d'or mythique de la «science pour la science » ou prétextant la rupture unilatérale du rapport de confiance entre État et universités pour refuser en bloc le processus de réforme, excommunier ceux qui s'y soumettent et constituer ce qui commence à ressembler à des « $\mathrm{Zad}$ » académiques, dans une accélération auto-réalisatrice des prophéties sur la constitution de collèges universitaires de seconde zone par aliénation des étages master-doctorat des établissements visés. Inversement, une tentation néoscientiste se dessine dans certains départements et laboratoires dont les responsables voudraient pouvoir saisir les opportunités du marché international de la connaissance sans plus être contraints par les missions sociales et sociétales d'un service public territorialisé à bout de souffle et de crédits.

À l'heure où le PIA3, initié en 2017, voit enfin les politiques publiques passer d'une ingénierie institutionnelle à une ingénierie des contenus, cherchant à stabiliser des systèmes cognitifs fondés sur la division du travail scientifique avec la création d'écoles universitaires de recherche thématisées (EUR), il serait désastreux que le monde académique se fracture sur l'enjeu de l'excellence, entre utopies réactionnaires et démissions éthiques. Cette crise n'est en effet pas seulement politique, elle apparaît de plus en plus également comme une crise épistémologique au sens où, justement, il en va de l'intelligence du monde dans la façon dont une société organise la mobilisation de son potentiel de formation et de recherche. Les universités françaises, qui ont su jusqu'alors préserver leur capacité à penser et à construire leur propre horizon scientifique et pédagogique, ne peuvent prendre le risque d'une guerre rhétorique entre «théologiens» et «utilitaristes», qui mènerait à l'affaiblissement de tous. Pour les replacer dans une dynamique de projet et d'émulation au service de l'esprit de découverte et valorisation, sans doute faudrait-il toutefois que les instances nationales et européennes des politiques de la recherche et de l'enseignement comprennent la rationalité propre de la recherche comme activité embarquée dans la vie sociale et environnementale d'un monde interconnecté et confronté à l'enjeu de sa propre soutenabilité. On éviterait ainsi de tuer dans son enfance la révolution cognitive initiée à la fin $\mathrm{du} \mathrm{xx}^{\mathrm{e}}$ siècle dans l'affirmation des méthodologies systémiques et relationnelles, voie de dépassement des limites du réductionnisme et de la division industrielle du travail scientifique.

Les universités françaises, moins ancrées que leurs homologues britanniques, allemandes ou italiennes dans une histoire urbaine et régionale longue, souffrent tout particulièrement de l'absence de mécanismes de compensation des effets délétères de la compétition scientifique. Ni l'esprit de corps des ingénieurs ni la loyauté envers l'institution des grands opérateurs de recherche appliquée ne viennent y compenser le struggle for life académique qui commence par le doctorat. De fait, les établissements et les communautés en difficulté dans la transition actuelle se sentent bien seuls dans leurs déboires actuels - pour ne rien dire de leurs enseignantschercheurs qui ne parviennent plus à donner cohérence à leurs tâches pédagogiques, administratives, de médiation et de recherche. Pour autant, on aurait tort de négliger la puissance symbolique et la richesse heuristique inentamées de l'idée d'Universitas, qui implique que ce que l'on fait à un seul membre de ce monde social, on le fait à l'ensemble; et ce que l'ensemble rend possible, chacun de ses membres peut le réaliser. Chacun passe ou ne passe pas le gué, mais le passage du gué est bien un enjeu pour l'ensemble-tant que cet ensemble existe et se reconnaît un idéal et un horizon communs.

Pour filer notre métaphore, c'est ainsi l'idée d'université en tant que telle qui succombera sur la rive ou qui franchira l'obstacle pour trouver une expression nouvelle dans l'écosystème du changement global. On peut certes à bon droit désespérer de la capacité des universités et des universitaires français à saisir cet enjeu. Mais on devrait peut-être aussi se demander si ce n'est pas de désespérer de leurs missions face aux injonctions contradictoires dont ils font l'objet qui les rend pour l'heure incapables d'accomplir les efforts nécessaires. L'élan libéral des années 1990, faut-il le rappeler, s'inscrivait dans l'horizon trompeur de la «mondialisation heureuse» et dans une acception irénique du potentiel des technosciences et des sciences du management à générer une société de marché toujours plus performante pour produire et pour partager des biens et des services en quantité croissante, en suivant l'exemple d'un monde académique états-unien qui, pourtant, commençait déjà à se déliter sous l'effet d'un utilitarisme sans limites. Dès le tournant de l'an 2000, Lawrence Busch $^{4}$ alertait sur les apories du «marché aux connaissances ».

Dans le contexte globalisé de 2018, dominé par la montée des périls géosystémiques et par la résistance acharnée des systèmes de pouvoirs issus du «monde du carbone », qui ont tout à perdre à l'entrée effective dans une nouvelle ère fondée sur une intelligence partagée des transitions nécessaires, il est bien davantage question de

\footnotetext{
${ }^{4}$ Busch L., 2000. The eclipse of morality. Science, State, and market, New York, Aldine de Gruyter; Busch L., 2014. Le marché aux connaissances. Néolibéralisme, enseignement et recherche, Versailles, Quæ. Voir aussi : Nowotny H., 2005. The public nature of science under assault. Politics, markets, science and the law, Berlin/New York, Springer.
} 
réencastrer l'économique dans le social, et les deux ensemble dans l'environnemental, dans la conception maîtrisée d'une nouvelle économie politique des ressources matérielles et immatérielles du monde. Il y a donc bien, pour les vieilles institutions universitaires, un gué à passer, mais sans doute pas celui de l'adaptation de la recherche et de la formation à une économie de la connaissance étroitement court-termiste et utilitariste d'ores et déjà condamnée. Réformer l'université demeure une ardente nécessité: non contre elle, mais avec elle, dans une acception renouvelée et réconciliée de la valeur de la «liberté» et de ses déclinaisons épistémologiques.

Citation de l'article : Cornu P., 2018. Les universités françaises au milieu du gué. Nat. Sci. Soc. 26, 3, 338-344. 